\title{
Intensive care unit versus non-intensive care unit postoperative management of head and neck free flaps: Comparative effectiveness and cost comparisons
}

\author{
Hassan Arshad, MD, ${ }^{1}$ Hatice Gulcin Ozer, PhD, ${ }^{2}$ Aaron Thatcher, MD, ${ }^{3}$ Matthew Old, MD, ${ }^{4}$ Enver Ozer, MD, ${ }^{4}$ Amit Agarwal, MD, ${ }^{4}$ Hosseinali Jafari, MD, ${ }^{5}$ \\ Danette Birkheimer, RN, ${ }^{6}$ Heidi Basinger, RN, ${ }^{6}$ L. Arrick Forest, MD, ${ }^{7}$ David E. Schuller, MD, ${ }^{4}$ Theodoros N. Teknos, MD ${ }^{4 *}$
}

\begin{abstract}
${ }^{1}$ Department of Head and Neck Surgery/Plastic and Reconstructive Surgery, Roswell Park Cancer Institute, Buffalo, New York, ${ }^{2}$ Biomedical Informatics Department, The Ohio State University Medical Center, Columbus, Ohio, ${ }^{3}$ Department of Otolaryngology - Head and Neck Surgery, University of Michigan, Ann Arbor, Michigan, ${ }^{4}$ Division of Head and Neck Oncology, Department of Otolaryngology - Head and Neck Surgery, The James Cancer Center, The Ohio State University Medical Center, Columbus, Ohio, ${ }^{5}$ Division of Otolaryngology - Head and Neck Surgery, Department of Surgery, Southern Illinois University, Springfield, Illinois, ${ }^{6}$ The James Cancer Center, Columbus, Ohio, ${ }^{7}$ Department of Otolaryngology - Head and Neck Surgery, The Ohio State University Medical Center, Columbus, Ohio.
\end{abstract}

Accepted 8 February 2013

Published online 18 June 2013 in Wiley Online Library (wileyonlinelibrary.com). DOl 10.1002/hed.23325

ABSTRACT: Background. Despite its widespread use, there is no consensus on the postoperative management in patients undergoing free flap reconstructions. We report the largest study comparing flap outcomes, morbidity, and cost in patients with head and neck cancer free flaps who recovered in the intensive care unit (ICU) versus a "specialty floor" setting.

Methods. This was a retrospective review of patients undergoing free flap surgery for head and neck defects over a 4-year period. Patients before a certain date went to the ICU for immediate postoperative care and after to a non-ICU setting. Postoperative medical and surgical complications and hospital charges were analyzed.
Results. Patients in the ICU group had a longer length of stay (LOS) and incurred greater hospital costs than the patients in the non-ICU setting. There was no difference in the flap failure rate between the 2 groups.

Conclusion. Consideration should be given to a floor-based postoperative management regimen for this patient population. (c) 2013 Wiley Periodicals, Inc. Head Neck 36: 536-539, 2014

KEY WORDS: free flap, microvascular reconstruction, head and neck, ICU, postoperative management

\section{INTRODUCTION}

Free flap microvascular surgery is an invaluable tool in reconstructing head and neck defects. Despite its widespread use, there is no consensus on the postoperative management in patients undergoing free flap reconstructions. Although much has been written about monitoring protocols and the effect of various pharmaceutical agents on platelet aggregation and flap survival, there is a paucity of literature regarding the effect of postoperative setting on outcome. A recent survey of academic otolaryngology-head and neck surgery departments in the United States showed that $88.9 \%$ of patients went directly to the intensive care unit (ICU) postoperatively, for an average of 2.4 days. $^{1}$ At many institutions, patients are kept sedated and intubated in the ICU to reduce the tension on the closure and anastomosis and, presumably, protect it from shearing forces imparted by patient movement. In other cases, patients go directly to the ICU because of lack of adequate nursing care in non-ICU settings. ${ }^{2}$

*Corresponding author: T. N. Teknos, 4024B Cramblett Hall, 456 W 10th Ave, Columbus, OH 43210. E-mail: ted.teknos@osumc.edu

This work was presented at the 8th International Conference on Head and Neck Cancer, Toronto, Canada, July 21-25, 2012.
Although the majority of patients undergoing free flap surgery go directly to the ICU, there are no good data to show that this is necessary to optimize flap survival and minimize morbidity. Several studies have illustrated that patients with head and neck cancer, in general, have equivalent outcomes whether they are managed postoperatively in an ICU or "specialty floor" setting. ${ }^{3-5}$ Similar studies comparing patient outcomes in a large cohort of free flap patients are lacking. A recently published retrospective review on a small group of patients $(n=52)$ showed no difference in flap outcomes when patients were extubated immediately after surgery compared to those extubated in the ICU. ${ }^{6}$ In this report, both groups went directly to the ICU but the group that was immediately extubated had a shorter ICU stay (2.0 vs 3.4 day; $p=.008)$ without a difference in hospital length of stay (LOS; 8.2 vs 9.5 days; $p=.21$ ). Interestingly, this study revealed that there was a higher incidence of pneumonia, use of physical restraints, and pharmacotherapy for delirium in the delayed extubation group. Taken together, the available literature on this subject would seem to imply that if adequate monitoring is available in a "specialty floor" setting, an ICU stay would not only be unnecessary but may actually result in a greater number of complications and higher costs to the health care system. In today's era of cost containment in health care, this relatively simple change in postoperative management could lead to substantial cost savings and improved outcomes. 
In this investigation, we report the largest study in the literature comparing flap outcomes, morbidity, and cost in patients with head and neck cancer free flap who recovered in the ICU versus a "specialty floor" setting.

\section{PATIENTS AND METHODS}

This was a retrospective review of patients undergoing free flap surgery for head and neck defects at the Arthur G. James Cancer Hospital at The Ohio State University from June 1, 2006, to June 30, 2010. Approval for the study was granted by the Ohio State University Institutional Review Board.

All patients before February 17, 2009 (ICU group) went straight to the ICU after surgery. The patients after that date (non-ICU) went straight to a "specialty specific floor.' A specialty specific floor was defined as a dedicated ward of the hospital where patients with head and neck cancer typically recover postoperatively. Before implementing the non-ICU protocol, extensive nursing education and training was required. This joint physician and nursing initiative included grand round presentations on patient management, use of monitoring equipment, identification of flap compromise, and development of protocols for nursing care of patients who underwent a free flap procedure. Although no new nurses needed to be hired, staffing levels were adjusted so that nurses caring for patients with flaps would be responsible for a maximum of 2 patients per shift. The only patients who went to the ICU after February 17, 2009, did so because their head and neck procedures included either a craniotomy or thoracotomy.

ICU protocol patients were taken directly to the ICU after surgery. Patients often remained sedated and on mechanical ventilation until deemed ready for weaning by the ICU and primary teams. The head and neck surgical staff instructed the ICU nurse as to the location and appearance of the flap and the appropriate Doppler signal. In the non-ICU protocol, after the patient came out of the operating room (OR), he/she went directly to the post-anesthesia care unit (PACU) off of mechanical ventilation. Then the floor nurse responsible for the patient's care would come to the PACU and assess the flap appearance and Doppler signal with the surgeons. The patient would then be transferred to the specialty floor after discharge from the PACU. Regardless of protocol, nurses checked the flap appearance and Doppler signal every hour for 48 hours, whereas residents checked it every 4 hours with Doppler and pinprick. After 48 hours, the flap was evaluated every 4 hours by the nurse and every 8 hours by the residents for an additional 2 days. Subsequent to this, the flap was checked once per shift by the nurses and twice daily by residents. All patients received daily aspirin.

Clinical data were collected for all patients from operative and anesthesia records, outpatient and inpatient charts, and nursing notes. Clinical data included age, sex, smoking status, alcohol use, previous therapy, stage, tumor type, site of cancer, flap type, operative time, and estimated blood loss. The comorbidities that were recorded included: diabetes, hypertension, history of stroke or myocardial infarction, pulmonary, renal, hepatic, vascular abnormalities, hypothyroidism, and nutritional status. All flap-related medical and surgical complications were recorded independently. Included in these were myocardial infarction, cerebrovascular accident, $\mathrm{O}_{2}$ desaturation $(<90 \%)$, ethanol withdrawal, return to the OR, transfer to ICU, fistula, cellulitis, wound dehiscence, abscess, sepsis, delirium, pneumonia, clostridium difficile colitis, and hematoma. Financial considerations for each patient included detailed analysis of hospital costs (direct and indirect, fixed and variable) from the OR, laboratory, pharmacy, nursing, consults, respiratory care, radiology, and supplies. All data were organized in a tabular format and analyzed using $\mathrm{R}$, a free software environment for statistical computing. The impact of each variable on outcome was tested using Wilcoxon rank-sum test for continuous variables, and the Fisher exact test for categorical variables. Results were considered significant if the $p<.05$.

\section{RESULTS}

A total of 257 patients were identified for this study. There were 119 identified in the ICU protocol (June 1, 2006 to February 17, 2009) and 138 patients who were operated on after February 17, 2009, and thus eligible for the non-ICU protocol. Of these 138 patients, 13 patients were ineligible for the non-ICU protocol as they were admitted to the ICU immediately after surgery because of coincident craniotomy and/or thoracotomy (ICU care was requested by the Thoracic Surgery and Neurosurgery Services). Therefore, 125 patients were included in the non-ICU protocol arm. The mean ages for the ICU and non-ICU protocol patients was 59.2 years and 58.9 years, respectively $(p=.74)$. The median number of comorbidities was 2 for each group. There was no statistical difference between the patients in each treatment arm when comparing previous therapies, age, sex, and tobacco or alcohol use (Table 1). There were no deaths in either cohort.

\section{Length of hospitalization}

When comparing protocols (Table 2), the patients in the ICU protocol group had a longer length of stay (LOS) than the patients in the non-ICU protocol (median of 9

TABLE 1. Characteristics of both patient groups (intensive care unit and non-intensive care unit protocol patients).

\begin{tabular}{lccc}
\hline & ICU protocol & Non-ICU protocol & $p$ value \\
\hline Age, mean & 59.2 & 58.9 & $.74^{*}$ \\
Comorbidities, mean & 2.53 & 2.24 & $.08^{*}$ \\
Sex & & & $.18^{\dagger}$ \\
$\quad$ Female & 35 & 48 & \\
$\quad$ Male & 84 & 77 & \\
Tobacco use & & & $.96^{\dagger}$ \\
$\quad$ Never & 21 & 23 & \\
$\quad$ Yes, currently & 52 & 56 & \\
$\quad$ Yes, quit in the past & 45 & 46 & $.30^{\dagger}$ \\
Alcohol use & & & \\
$\quad$ Never & 47 & 58 & \\
$\quad$ Yes & 72 & 67 & \\
\hline
\end{tabular}

Abbreviation: ICU, intensive care unit.

${ }^{*}$ Wilcoxon rank-sum test.

${ }^{\dagger}$ Fisher exact test. 
TABLE 2. Outcome comparisons between patients taken directly to the floor versus intensive care unit in the postoperative setting.

\begin{tabular}{lccc}
\hline Variables & $\begin{array}{c}\text { ICU } \\
\text { protocol }(n=119)\end{array}$ & $\begin{array}{c}\text { non-ICU protocol } \\
(n=125)\end{array}$ & $p$ value \\
\hline Median LOS, d & 9 & 8 & .008 \\
Complete flap failure & $3.4 \%(4 / 119)$ & $4 \%(5 / 125)$ & 1 \\
Blood transfusion & $13.4 \%(16 / 119)$ & $5.6 \%(7 / 125)$ & .047 \\
\hline
\end{tabular}

Abbreviations: ICU, intensive care unit; LOS, length of stay.

days vs 8 days; mean, 10.28 vs $9.89 ; p=.008)$. The average LOS in the ICU was 3.5 days. When comparing all patients who went directly to the ICU during the entire study period (includes ICU protocol patients plus craniotomy/thoracotomy patients) to those who went directly to the floor (non-ICU), ICU patients had a statistically longer LOS compared to non-ICU patients (median of 9 days vs 8 days; $p=.007$; Table 3 ).

After ICU vs non-ICU status, the next most important determinant of the length of hospitalization was the presence of postoperative complications. The median number of complications was 1 for both ICU and non-ICU patients $(p=.67)$. However, the LOS in patients who had a complication postoperatively versus those with no complication was highly significant (median of 8 days vs 10 days; $p<.00001$ ). Eleven patients (11 of $124 ; 8.8 \%$ ) in the non-ICU protocol were secondarily transferred to the ICU because of flap failure $(n=5), \mathrm{O}_{2}$ desaturation below $90 \%(n=4)$, or other postoperative complications $(n=2)$. When taking all factors into account, the independent predictor of transfer to the ICU after being on the floor was an estimated operative blood loss of $>500 \mathrm{cc}(p=.027)$. There were no significant differences in the complication rate of either group with regard to cardiac, neurologic, or other organ system disease. The ICU group had more pulmonary complications than the non-ICU group $(p=.002)$.

\section{Flap outcomes}

When comparing ICU to non-ICU protocol patients, there was no statistical difference in complete flap failure rate ( 4 of $119 ; 3.4 \%$ vs 5 of $125 ; 4 \% ; p=1$ ). Because there were some patients who needed an ICU bed after surgery (for medical reasons or for neurologic monitoring), patients who went straight to the ICU regardless of protocol were compared to those who went straight to the floor (non-ICU) regardless of protocol. There was also no difference in the complete flap failure rate between the ICU and non-ICU patients (4 of $131 ; 3.1 \%$ vs 5 of 126; $4 \% ; p=.74)$. Analysis of the entire study population revealed that flap loss (complete or partial) was associated with salvage surgery. Flap loss (complete or partial) was rare in patients without previous therapy (5 of 149; $3.3 \%$ ), but was much more prevalent in patients who had undergone previous extirpative surgery alone (6 of 41; $14.6 \%)$ or previous radiation/chemoradiation $+/$ - surgery (8 of $67 ; 11.9 \%$ ). This was highly statistically significant at $p=.006$. Overall, $7.6 \%$ of patients were taken back to the OR either for flap salvage or management of a surgical complication with no difference noted between the ICU and non-ICU groups.

\section{Cost analysis}

Financial comparisons were drawn between the ICU and non-ICU groups including total charges, revenue, and costs incurred for each patient in the OR, laboratory, pharmacy, nursing, consults, respiratory care, radiology, and supplies. Increases in PACU usage in the non-ICU were also accounted for. Although the ICU group had statistically significant longer operative times $(p=.017)$, the overall cost for OR services in the non-ICU group was higher because of increased PACU costs. There were no additional costs in terms of nurse training. Nursing assignments were altered to accommodate the patients who underwent a free flap procedure. The average net revenue per patient in the ICU portion of the protocol was $\$ 2134$ greater than the average revenue per patient in the non-ICU protocol. Interestingly, however, the average cost per patient was also $\$ 3238$ higher in the ICU protocol patients. As a result, the average profit per patient or contribution to the margin was \$1104 higher in patients who were managed postoperatively in a non-ICU setting. Cost savings per patient included the following: laboratory (\$835), pharmacy (\$651), ICU nursing (\$4881), respiratory care (\$656), radiology (\$249), and supplies (\$224). This cost savings reflects the increased cost of ICU management with daily chest X-rays, blood draws, ventilator management, specialized nursing, and pharmaceutical costs for sedation. Therefore, non-ICU management of free flap patients postoperatively not only results in equivalent quality of care and shorter LOS, but also contributes more revenue to the hospital margin.

\section{DISCUSSION}

Free flap reconstructive surgery allows head and neck surgeons to perform radical resections while minimizing cosmetic and functional defects. Even though it is widely used, the postoperative management varies among institutions. Some of these differences involve the way in which the flap is monitored, which pharmacologic agents are used to improve flap blood flow, and where patients are admitted after the OR. In many institutions, free flap patients go to the ICU after surgery. A 2007 survey showed this to be the case in almost $90 \%$ of patients undergoing free flap reconstructive surgery for head and neck defects. The average LOS in the ICU for free flap patients that is reported in the literature varies from 2 to 11 days. The advantages cited in ICU admission included relative immobilization limiting shearing forces on the vascular anastomosis, closer flap monitoring, and optimal blood pressure control, among others. However, there are numerous disadvantages with ICU monitoring, including

TABLE 3. Comparison of patients who went straight to the intensive care unit after surgery and patients going straight to the floor.

\begin{tabular}{lccc}
\hline Variables & $\begin{array}{c}\text { ICU patients } \\
(n=131)\end{array}$ & $\begin{array}{c}\text { Non-ICU patients } \\
(n=126)\end{array}$ & $p$ value \\
\hline Median LOS, $d$ & 9 & 8 & .007 \\
Complete flap failure & $3.1 \%(4 / 131)$ & $4 \%(5 / 126)$ & .74 \\
Blood transfusion & $12.2 \%(16 / 131)$ & $5.6 \%(7 / 125)$ & .08 \\
\hline
\end{tabular}

Abbreviations: ICU, intensive care unit; LOS, length of stay. 
the use of sedation, which can result in decreased blood pressure compromising flap perfusion, as well as prolonged ventilation leading to pneumonia and increased hospital costs. The current study is the largest and first in the literature to illustrate that there are many advantages to non-ICU management of free flap patients compared to routine ICU management.

The most important finding in this study is that there was no significant increased morbidity or mortality in patients who went directly to a specialty floor compared to those who went directly to the ICU. This can only be accomplished, however, when there is a well-established, highly trained, and motivated nursing team that understands the nuances of patients with head and neck free flap management. At the Arthur G. James Cancer Hospital, a collaborative teaching effort on behalf of both the surgical staff and nursing leadership was put in place before routinely admitting postoperative free flap patients to the floor. This team effort not only resulted in superior patient care but it also instilled a tremendous sense of ownership and responsibility to the nursing staff. Other important findings in this study were that patients who were admitted directly to the floor not only had equivalent quality of care and outcomes, but also had significantly shorter hospitalizations than their counterparts who were admitted to the ICU postoperatively. This relatively simple change in practice also resulted in lower hospital costs and a greater profit margin for the institution. In an institution that does at least 100 free flaps per year, the overall cost savings is over $\$ 100,000$. Some of these savings may manifest if the patients are sent to the ICU unsedated and off mechanical ventilation, however, nonICU management also saves valuable ICU space for other patients.

We acknowledge some limitations in this study. First, it is retrospective and not a randomized trial. Second, because the non-ICU group was more recent in time, there may have been an overall increase in the quality of postoperative care as experience grew with these patients. Finally, there were additional primary and flap surgeons in the non-ICU group.

Before this investigation, there were few other studies in the literature that have attempted to compare flap outcomes and postoperative morbidity in this patient population. Godden et $\mathrm{al}^{3}{ }^{3}$ illustrated that, in general, patients with head and neck cancer can as effectively be managed on a specialty floor as the ICU. This study, however, did not specifically differentiate between flap and non-flap patients. McVeigh et $\mathrm{al}^{7}$ showed a $98 \%$ flap survival rate in 68 patients undergoing free flap reconstructions for oral and oropharyngeal defects who were monitored outside the ICU setting. This illustrated that non-ICU management of these complex patients was feasible and safe but their study did not include an ICU comparison group. Recently, the University of Virginia group examined whether immediate postoperative extubation would affect free flap outcomes and morbidity in head and neck free flap patients. ${ }^{6}$ In this study, all patients were taken to the ICU but 26 of 52 patients were extubated immediately after surgery. They found no differences in terms of flaprelated complications when compared to the group that remained intubated for 24 hours. There was a significant increase in the rate of postoperative pneumonia, delirium, and the use of restraints in the late extubation group, however. The closest study to ours in the literature was conducted in the United Kingdom. In a much smaller cohort, they found no difference in general medical complications or flap survival regardless of where free flap patients were taken immediately after surgery. ${ }^{8}$

Taken together, the available literature and the current investigation question the rationale for sending patients directly to the ICU after head and neck free flap surgery. It is clear that it is unnecessary to keep patients sedated and on mechanical ventilation solely for purposes of flap survival. However, there are several instances in which ICU management is clearly indicated. This includes cases in which much more aggressive hemodynamic or neurologic monitoring is indicated, such as cases in which a thoracotomy, laparotomy, or craniotomy were performed. In this study, we also recognized that patients who had greater than $500 \mathrm{cc}$ of operative blood loss were at a significantly higher risk for transfer from the floor to the ICU in the postoperative setting. As a result of this finding, these patients may be best served with closer monitoring in the ICU setting after head and neck free flap surgery.

\section{CONCLUSION}

In this study, patients with head and neck cancer undergoing resection and immediate free flap reconstruction had equivalent outcomes with regard to flap survival and morbidity whether they were taken directly to the ICU or to a specialized hospital ward. Additional advantages of having patients go directly to the floor include significantly shorter hospitalization and significantly lower hospital costs. Patients who should be monitored postoperative in the ICU include those who have greater than 500 cc of operative blood loss and those who undergo thoracotomy, craniotomy, or laparotomy as part of their ablative procedure.

\section{REFERENCES}

1. Spiegel JH, Polat JK. Microvascular flap reconstruction by otolaryngologists: prevalence, postoperative care, and monitoring techniques. Laryngoscope 2007; 117:485-490

2. Haddock NT, Gobble RM, Levine JP. More consistent postoperative care and monitoring can reduce costs following microvascular free flap reconstruction. J Reconstr Microsurg 2010;26:435-439.

3. Godden DR, Patel M, Baldwin A, Woodwards RT. Need for intensive care after operations for head and neck cancer surgery. Br J Oral Maxillofac Surg 1999;37:502-505.

4. To EW, Tsang WM, Lai EC, Chu MC. Retrospective study on the need of intensive care unit admission after major head and neck surgery. ANZ $J$ Surg 2002;72:11-14.

5. Nkenke E, Vairaktaris E, Stelzle F, Neukam FW, St Pierre M. No reduction in complication rate by stay in the intensive care unit for patients undergoing surgery for head and neck cancer and microvascular reconstruction. Head Neck 2009;31:1461-1469.

6. Allak A, Nguyen TN, Shonka DC Jr, Reibel JF, Levine PA, Jameson MJ Immediate postoperative extubation in patients undergoing free tissue transfer. Laryngoscope 2011;121:763-768.

7. McVeigh KP, Moore R, James G, Hall T, Barnard N. Advantages of not using the intensive care unit after operations for oropharyngeal cancer: an audit at Worcester Royal Hospital. Br J Oral Maxillofac Surg 2007;45: 648-651.

8. McMillian WD, Taylor S, Lat I. Sedation, analgesia, and delirium in the critically ill patient. J Pharm Pract 2011;24:27-34. 